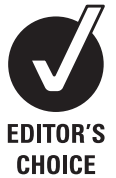

CHOICE
${ }^{1}$ Cardiovascular Center, Andreas Gruntzig Cardiac Catheterization Laboratories, University Hospital Zurich, Zurich, Switzerland

${ }^{2}$ Cardiocentro Ticino, Lugano, Switzerland

${ }^{3}$ Cardiovascular Center, Echocardiography, Zurich, Switzerland

${ }^{4}$ Department of Anesthesiology, University Hospital Zurich, Zurich, Switzerland

${ }^{5}$ Cardiovascular Surgery, Cardiovascular Center, University Hospital Zurich, Zurich, Switzerland

Correspondence to Professor Roberto Corti, Andreas Gruntzig Laboratories, Cardiovascular Center, University Hospital Zurich, Ramistrasse 100, 8091 Zurich Switzerland; roberto.corti@usz.ch

Accepted 17 August 2011 Published Online First 7 October 2011

\title{
Acute haemodynamic changes after percutaneous mitral valve repair: relation to mid-term outcomes
}

\author{
Oliver Gaemperli, ${ }^{1}$ Marco Moccetti, ${ }^{1,2}$ Daniel Surder, ${ }^{1,2}$ Patric Biaggi, ${ }^{3}$ David Hurlimann, ${ }^{3}$ \\ Oliver Kretschmar, ${ }^{1}$ Ines Buehler, ${ }^{1}$ Dominique Bettex, ${ }^{4}$ Christian Felix, ${ }^{4}$ Thomas F Luscher, \\ Volkmar Falk, ${ }^{5}$ Jurg Grunenfelder, ${ }^{5}$ Roberto Corti ${ }^{1}$
}

\section{ABSTRACT}

Background Percutaneous mitral valve repair (MVR) using the Evalve MitraClip has been recently introduced as a potential alternative to surgical MVR.

Objective To assess immediate haemodynamic changes after percutaneous MVR using right heart catheterisation.

Design Single-centre longitudinal cohort study.

Setting Tertiary referral centre.

Patients Fifty consecutive non-surgical patients lage $74 \pm 14$ years, EuroSCORE $26 \pm 14$ ) with moderate to severe $(3+)$ and severe $(4+)$ mitral regurgitation (MR) due to functional $(56 \%)$, degenerative $(30 \%)$ or mixed (14\%) disease were selected.

Interventions MitraClip implantation was performed under general anaesthesia with fluoroscopy and echocardiographic guidance. Haemodynamic variables were obtained before and after MVR using standard right heart catheterisation and oximetry.

Main outcome measures Haemodynamic changes immediately before and after MVR.

Results Acute procedural success (reduction in MR to grade $2+$ or less) was achieved in 46 (92\%) patients. Mitral valve clipping reduced mean pulmonary capillary wedge pressure (mPCWP) (from 17 \pm 7 to $12 \pm 5 \mathrm{~mm} \mathrm{Hg}$ ), PCWP v-wave (from $24 \pm 11$ to $16 \pm 7 \mathrm{~mm} \mathrm{Hg}$ ) and mean pulmonary artery pressure (mPAP) (from $29 \pm 12$ to $24 \pm 6 \mathrm{~mm} \mathrm{Hg}$ ), and increased the cardiac index (Cl) (from $3.1 \pm 1.0$ to $3.9 \pm 1.1 \mathrm{l} / \mathrm{min}$ / $m^{2}$ ) (all $\left.p<0.05\right)$. On Cox univariate regression analysis, mPCWP, PCWP v-wave- and mPAP-changes were associated with death, open-heart surgery for MR and/or hospitalisation for heart failure on follow-up.

Conclusion In a heterogeneous population with predominantly functional MR, percutaneous MVR with the Evalve MitraClip system lowers mPCWP, PCWP v-wave and mPAP by $20 \%, 20 \%$ and $8 \%$, respectively, and increases the $\mathrm{Cl}$ by $32 \%$.

\section{INTRODUCTION}

The management of severe mitral regurgitation $(\mathrm{MR})$ in patients at high surgical risk often represents a significant clinical challenge as clinicians have to weigh carefully the risks of open-heart surgery against the potential clinical benefit of mitral valve repair (MVR). The preferred surgical option in patients with degenerative $M R$ is MVR owing to its favourable clinical outcome compared with mitral valve replacement. ${ }^{1} 2$ However, in patients with functional MR, evidence in favour of MVR is less well established. ${ }^{3}{ }^{4}$ A percutaneous method for MVR has been developed. ${ }^{5}$ This technique is based on the surgical method developed by Alfieri and allows edge-to-edge approximation of the middle scallops of the mitral valve leaflets by percutaneous delivery of a mitral clip, thereby creating a double-orifice mitral valve. ${ }^{6}$ Initial clinical experience in humans shows that percutaneous edge-to-edge MVR can be performed safely and allows durable reduction of $M R$ severity. ${ }^{7-10}$ A recent randomised comparison with surgical mitral valve reconstruction or replacement showed that the percutaneous technique was safer, albeit with a slightly lower efficacy. ${ }^{11}$

In previously published reports, haemodynamic success of mitral clip implantation has traditionally been assessed using Doppler echocardiographic parameters. However, assessment of MR parameters after clip implantation can be challenging, subject to operator experience and is associated with a considerable interobserver variability. ${ }^{12}$ Additionally, procedural success of mitral clip implantation does not necessarily translate into haemodynamic improvement of cardiac performance. To date, limited data on the impact of percutaneous MVR on objective haemodynamic parameters from cardiac catheterisation are available. ${ }^{13}$ Moreover, previously published reports had stringent inclusion criteria for valve morphology, left ventricular (LV) dimensions and function, and operability, and therefore potentially excluded a substantial number of patients who might have benefited from this procedure. ${ }^{7}$ We therefore investigated haemodynamic parameters before and after mitral clip implantation obtained from invasive cardiac catheterisation in patients at high surgical risk undergoing percutaneous edge-to-edge MVR.

MATERIALS AND METHODS Patient population and study design

The study included consecutive patients undergoing percutaneous MVR at the University Hospital of Zurich, Switzerland. Indications for treatment of $M R$ and individual surgical risk were assessed by an interdisciplinary team of interventional cardiologists, echocardiographers, cardiac anaesthetists and cardiac surgeons. Patients were selected for the procedure if they had moderate-tosevere $(3+)$ or severe $(4+) \mathrm{MR}$ of functional or 
degenerative origin as graded according to the recommendations of the American Society of Echocardiography (ASE), ${ }^{12}$ and met class I or Ila indications for mitral valve surgery as recommended by the 2006 American College of Cardiology (ACC)/American Heart Association (AHA) guidelines for management of patients with valvular heart disease. ${ }^{14}$ A key additional inclusion criterion was a high surgical risk defined by a logistic European System for Cardiac Operative Risk Evaluation (EuroSCORE) ${ }^{15}$ mortality $>20 \%$ or by the presence of specific surgical risk factors not covered by the EuroSCORE (ie, frailty, immunosuppressive therapy). Exclusion criteria were rheumatic heart disease, endocarditis, a mitral valve orifice area $\leq 2.0 \mathrm{~cm}^{2}$, extensive prolapse of flail leaflets (prolapse width $>25 \mathrm{~mm}$, flail gap $>20 \mathrm{~mm}$ ) or any interventional or surgical procedure within 30 days of the index procedure. The study protocol was approved by the local institutional review board and all patients gave written informed consent.

\section{MVR procedure}

The percutaneous MVR procedure was performed under general anaesthesia with fluoroscopy and three-dimensional transoesophageal echocardiographic (3D TOE) guidance using the MitraClip device (Evalve Inc, Menlo Park, California, USA) via a femoral venous approach. The procedure was performed under general anaesthesia (because of the need for TOE) with a continuous infusion of intravenous propofol and remifentanil. Patients were ventilated routinely with an inspiratory oxygen fraction of $80 \%$, which was maintained over the entire procedure. After trans-septal puncture, the trans-septal sheath was exchanged by a steerable $24 \mathrm{~F}$ guide catheter through which the clip delivery system was advanced into the left atrium. Thereafter, the MitraClip device was manoeuvred into the left ventricle and aligned with the origin of the regurgitant jet using prespecified 3D TOE views, as previously described. ${ }^{16}$ Once the correct position was assured, the clip was pushed below the level of the mitral leaflets. After opening the two arms of the clip, the device was retracted until both leaflets were grasped and was then closed to approximate both scallops and restore coaptation. Careful consideration was given to a perpendicular orientation of the clip arms with regard to the leaflet edges before closing the clip. Mitral valve function and reduction of MR was immediately assessed by $3 \mathrm{D}$ TOE and haemodynamic measurements. If necessary, the device could be repositioned by reopening the arms and releasing the leaflets. Acute procedural success (APS) was defined as successful MitraClip implantation with MR reduction to grade $2+$ or less. If APS was achieved, the clip was deployed and the delivery system withdrawn. Repeat clip insertion could be performed if the haemodynamic result was inadequate after the first clip had been implanted.

\section{Invasive haemodynamic data}

Invasive haemodynamic monitoring (continuous tracings of LV filling pressures (LV end-diastolic pressure (LVEDP)) and systolic arterial pressure) was conducted during the entire procedure by retrograde placement of a $5 \mathrm{~F}$ pigtail catheter into the left ventricle. Before and immediately after percutaneous MVR, right heart catheterisation was performed using a $6 \mathrm{~F}$ singlelumen, balloon-tipped, flow-directed Swan-Ganz catheter (Arrow International, Inc, Reading, Pennsylvania, USA) to obtain pulmonary capillary wedge pressure (PCWP), pulmonary artery pressure (PAP), pulmonary artery oxygen saturation and right atrial pressure (RAP). Systemic arterial oxygen saturation was obtained from the LV pigtail catheter. Left atrial pressure (LAP) was measured through the trans-septal sheath. Cardiac output (CO) and cardiac index (CI) were calculated by the Fick method. ${ }^{17}$ Systemic (SVR) and pulmonary vascular resistance (PVR) were calculated as the ratio between the pressure drop along the vascular bed and the cardiac output and converted in metric units $\left(\mathrm{dyn} \times \mathrm{s} \times \mathrm{cm}^{-5}\right)$. A baseline haemodynamic study in a conscious non-sedated state was available in 39 (78\%) patients to document the effects of general anaesthesia on haemodynamic values before the MVR procedure (median (IOR) time interval 19 (4-43) days).

\section{Follow-up}

Clinical follow-up was obtained at 3, 6 and 12 months after percutaneous MVR including assessment of New York Heart Association (NYHA) functional status. Adverse cardiovascular events included death, rehospitalisation for congestive heart failure $(\mathrm{CHF})$ and surgical reoperation for MR. Severity of residual $M R$ was assessed by transthoracic echocardiography 3 months after the MVR procedure and graded according to ASE recommendations, ${ }^{12}$ and $\mathrm{LV}$ volumes, LV ejection fraction and left atrial (LA) diameter (on parasternal M-mode) were recorded.

\section{Statistical analysis}

Statistical analysis was performed using the SPSS software package (SPSS 12.0.1 for Windows, SPSS Corp). Quantitative data are expressed as mean \pm SD and categorical data given as proportions and percentages. Statistical comparison of quantitative data was performed using a paired two-tailed Student $t$ test. Comparison of categorical data was conducted with Fisher's exact test. Survival free of major cardiovascular events was analysed using the Kaplan-Meier method. The predictive value of haemodynamic parameters for outcome variables was assessed using receiver operating characteristics analysis. Additionally, univariate Cox regression analysis was employed to identify haemodynamic predictors of events and the results given as $\mathrm{HR}$ with their respective $95 \%$ CIs. A p value $<0.05$ was considered statistically significant for all tests.

\section{RESULTS}

\section{Patient population}

Between May 2009 and December 2010, 50 consecutive patients underwent percutaneous MVR at our institution. The mean age was $74 \pm 14$ years and 18 (36\%) were female. The clinical characteristics of our study population are given in table 1 . All patients had a history of CHF and $43(86 \%)$ patients were in NYHA functional class III-IV. Estimated surgical risk was high with a EuroSCORE mortality, STS mortality, and STS mortality and morbidity score of $26 \pm 14$ (range $8-73$ ), $7 \pm 6 \%$ (range $0-35 \%$ ) and $35 \pm 19 \%$ (range $8-76 \%$ ), respectively. Sixteen $(32 \%)$ patients had a EuroSCORE $<20$ but were considered high risk for surgery owing to previous complex cardiac or aortic surgery $(n=5)$, frailty $(n=7)$, excessive alcohol consumption $(n=1)$, extreme obesity $(n=1)$, cancer $(n=1)$, multiple sclerosis $(n=1)$, immunosuppressive therapy $(n=1)$, thrombocytopenia $(n=1)$, or because of refusal to undergo surgery $(n=2)$. The aetiology of $\mathrm{MR}$ is shown in table 2. A functional mechanism was identified in $28(56 \%)$ of patients, whereas a degenerative aetiology was present in $15(30 \%)$ patients.

\section{MVR procedure}

The results of the MVR procedure and in-hospital outcomes are given in table 3. APS was achieved in 46 (92\%) patients. Failed procedures were encountered in three patients with functional and one patient with mixed MR. Reasons for procedural failure were inability to properly grasp both leaflets in a patient with 
Table 1 Patient characteristics $(n=50)$

\begin{tabular}{ll}
\hline Age, years & $74 \pm 14$ \\
Female gender, $\mathrm{n}(\%)$ & $18(36)$ \\
BMI (kg/m²) & $25 \pm 5$ \\
Clinical features, n (\%) & \\
Arterial hypertension & $33(66)$ \\
Hyperlipidaemia & $20(40)$ \\
Diabetes mellitus & $7(14)$ \\
Coronary artery disease & $19(38)$ \\
$\quad$ Previous MI & $14(28)$ \\
Previous PCI & $12(24)$ \\
Previous CABG & $7(14)$ \\
Left ventricular ejection fraction (\%) & $47 \pm 18$ \\
Previous valve surgery & $4(8)$ \\
Previous vascular/thoracic surgery & $2(4)$ \\
Atrial fibrillation & $33(66)$ \\
COPD & $7(14)$ \\
Previous stroke & $4(8)$ \\
Impaired renal function & $28(56)$ \\
Cancer & $2(4)$ \\
NYHA functional class, n (\%) & \\
II & $7(14)$ \\
III & $34(68)$ \\
IV & $9(18)$ \\
Surgical risk & \\
Logistic EuroSCORE, \% & \\
STS mortality score, \% & $26 \pm 14$ \\
STS mortality and morbidity score, \% & $7 \pm 6$ \\
\hline Data are given as mean \pm SD unless otherwise stated. \\
BMl, body mass index; CABG, coronary artery bypass grafting; COPD, \\
chronic obstructive pulmonary disease; EuroSCORE, European System \\
for Cardiac Operative Risk Evaluation; MI, myocardial infarction; NYHA, \\
New York Heart Association; PCI, percutaneous coronary intervention; \\
STS, Society of Thoracic Surgeons. \\
& $35 \pm 19$ \\
\hline
\end{tabular}

a commissural flail $(n=1)$, inability to deploy the clip at the target region owing to massive left atrial enlargement $(n=1)$, inappropriate visualisation of the posterior leaflet owing to annular calcification $(\mathrm{n}=1)$ and procedure-related chordal rupture with secondary aggravation of $M R(n=1)$. There were no procedural deaths. The patient with the massive LA dilatation died on the 15th post-interventional day owing to severe congestive heart failure and was the only in-hospital death reported in our series.

Overall, complications from mitral clipping procedures were rare (table 3) and length of stay in high-dependency units was short. Two patients (4\%) underwent immediate closure of iatrogenic atrial septal defect using an Amplatzer occluder and two patients (4\%) required intra-aortic balloon counterpulsation owing to haemodynamic instability during the procedure (both patients with procedural failure). None of the patients

Table 2 Aetiology and mechanism of mitral regurgitation $(n=50)$

\begin{tabular}{lc}
\hline Functional & $28(56)$ \\
Ischaemic & $12(24)$ \\
Non-ischaemic & $16(32)$ \\
Degenerative & $15(30)$ \\
Prolapse/flail posterior leaflet only & $7(14)$ \\
Prolapse/flail anterior leaflet only & $3(6)$ \\
Bi-leaflet prolapse/flail & $5(10)$ \\
Mixed & $7(14)$ \\
\hline Data are given as absolute numbers and percentages.
\end{tabular}

Data are given as absolute numbers and percentages.
Table 3 Procedure-related data

\begin{tabular}{lc}
\hline APS, $\mathrm{n}(\%)$ & $46(92)$ \\
Number of clips implanted, $\mathrm{n}(\%)$ & \\
1 & $18(36)$ \\
2 & $28(56)$ \\
3 & $4(8)$ \\
CCU/ICU stay (days) & $1(0-23)$ \\
Mechanical ventilation $>48 \mathrm{~h}, \mathrm{n}(\%)$ & $0(0)$ \\
IABP, $\mathrm{n}(\%)$ & $2(4)$ \\
Complications from trans-septal puncture, & $2(4)$ \\
$\mathrm{n}(\%)$ & \\
PM/CRT/ICD lead displacement, $\mathrm{n}(\%)$ & $0(0)$ \\
Clip-related chordal rupture, $\mathrm{n}(\%)$ & $1(2)$ \\
In hospital infection, $\mathrm{n}$ (\%) & $0(0)$ \\
In-hospital congestive heart failure, $\mathrm{n}$ (\%) & $5(10)$ \\
In-hospital arrhythmia, $\mathrm{n}(\%)$ & $1(2)$ \\
Early mitral valve surgery, $\mathrm{n}$ (\%) & $2(4)$ \\
In-hospital death, $\mathrm{n}(\%)$ & $1(2)$ \\
Hospital stay (days) & $5(1-60)$ \\
\hline *Data are given as median (range) or absolute numbers and percentages. \\
APS, acute procedural success (defined as successful MitraClip \\
placement with MR reduction to grade 2 or less); CCU, coronary care \\
unit; CRT, cardiac resynchronisation therapy; IAB, intra-aortic balloon \\
pump; ICD, implantable cardioverter-defibrillator; ICU, intensive care unit; \\
PM, pacemaker.
\end{tabular}

had access-site complications, bleeding requiring transfusion, pacemaker lead displacement, or in-hospital infection.

\section{Haemodynamic data}

Invasive haemodynamic data were available at baseline (during the screening phase) in a conscious non-sedated state in 39 (78\%) patients and during the MVR procedure immediately before and after MitraClip deployment in all 50 patients.

Immediate haemodynamic changes during the MVR procedures are shown in table 4 (values obtained under general anaesthesia during the index procedure immediately before and after MVR). Mitral valve clipping significantly reduced mean SVR (from $985 \pm 414$ to $791 \pm 255 \mathrm{dyn} \times \mathrm{s} \times \mathrm{cm}^{-5}$ ), mean PCWP (from $17 \pm 7$ to $12 \pm 5 \mathrm{~mm} \mathrm{Hg}$ ), PCWP v-wave (from $24 \pm 11$ to $16 \pm 7 \mathrm{~mm} \mathrm{Hg}$ ), mean LAP (from $16 \pm 7$ to $12 \pm 5 \mathrm{~mm} \mathrm{Hg}$ ), LAP $\mathrm{v}$-wave (from $27 \pm 14$ to $18 \pm 10 \mathrm{~mm} \mathrm{Hg}$ ) and mean PAP (from $29 \pm 12$ to $24 \pm 6 \mathrm{~mm} \mathrm{Hg}$ ). There was no change in mean arterial

Table 4 Invasive haemodynamic data*

\begin{tabular}{lccc}
\hline & $\begin{array}{l}\text { Before MVR } \\
(\mathbf{n = 5 0 )}\end{array}$ & $\begin{array}{l}\text { After MVR } \\
(\mathbf{n}=\mathbf{5 0 )}\end{array}$ & p Value \\
\hline Mean RAP (mm Hg) & $8 \pm 4$ & $8 \pm 3$ & 0.67 \\
Mean PAP (mm Hg) & $29 \pm 12$ & $24 \pm 6$ & 0.013 \\
Mean PCWP (mm Hg) & $17 \pm 7$ & $12 \pm 5$ & 0.002 \\
PCWP v-wave (mm Hg) & $24 \pm 11$ & $16 \pm 7$ & 0.003 \\
Mean LAP (mm Hg) & $16 \pm 7$ & $12 \pm 5$ & 0.014 \\
LAP V-wave (mm Hg) & $27 \pm 14$ & $18 \pm 10$ & 0.011 \\
LVEDP (mm Hg) & $15 \pm 5$ & $15 \pm 6$ & 0.68 \\
MAP (mm Hg) & $71 \pm 17$ & $72 \pm 11$ & 0.85 \\
CO (1/min) & $5.4 \pm 1.7$ & $7.0 \pm 1.8$ & $<0.001$ \\
CI (I/min $\left./ \mathrm{m}^{2}\right)$ & $3.1 \pm 1.0$ & $3.9 \pm 1.1$ & $<0.001$ \\
SVR (dyn $\left.\times \mathbf{s} \times \mathrm{cm}^{-5}\right)$ & $985 \pm 414$ & $791 \pm 255$ & 0.021 \\
PVR (dyn $\left.\times \mathbf{s} \times \mathrm{cm}^{-5}\right)$ & $198 \pm 178$ & $143 \pm 67$ & 0.054 \\
\hline
\end{tabular}

Data are given as mean $\pm S D$.

*All haemodynamic values were recorded under general anaesthesia during the index session, that is, immediately before and immediately after concluding the procedure (MVR). $\mathrm{Cl}$, cardiac index; CO, cardiac output; LAP, left atrial pressure; LVEDP, left ventricular enddiastolic pressure; MAP, mean arterial pressure; MVR, mitral valve repair; PAP, pulmonary artery pressure; PCWP, pulmonary capillary wedge pressure; PVR, pulmonary vascular resistance; RAP, right atrial pressure; SVR, systemic vascular resistance. 
pressure, LVEDP and RAP after MVR. A borderline significant decrease in PVR was seen after MVR. Conversely, CO (from $5.4 \pm 1.7$ to $7.0 \pm 1.8 \mathrm{l} / \mathrm{min}$ ) and CI (from $3.1 \pm 1.0$ to $3.9 \pm 1.1 \mathrm{l} /$ $\mathrm{min} / \mathrm{m}^{2}$ ) increased after mitral clipping. Average percentage decreases in mean PAP, mean PCWP and PCWP v-wave were $8 \pm 22 \%, 20 \pm 33 \%$ and $20 \pm 40 \%$, respectively and the increase in CI was $32 \pm 39 \%$ (figure 1). Figure 2 gives an example of a representative patient before and after mitral clipping.

Of note, haemodynamic data obtained at baseline (screening phase) in a conscious non-sedated state differed markedly from values measured under general anaesthesia immediately before the MVR procedure. While RAP $(9 \pm 5 \mathrm{~mm} \mathrm{Hg})$ and LVEDP $(18 \pm 7 \mathrm{~mm} \mathrm{Hg})$ were comparable, mean arterial pressure $(84 \pm 15 \mathrm{~mm} \mathrm{Hg})$, mean PAP $(37 \pm 13 \mathrm{~mm} \mathrm{Hg})$, mean PCWP $(23 \pm 8 \mathrm{~mm} \mathrm{Hg})$, SVR $\left(1647 \pm 537 \mathrm{dyn} \times \mathrm{s} \times \mathrm{cm}^{-5}\right)$ and PVR $\left(343 \pm 299\right.$ dyn $\left.\times \mathrm{s} \times \mathrm{cm}^{-5}\right)$ were higher and CO $(4.0 \pm 1.4 \mathrm{l} / \mathrm{min})$ and CI $\left(2.2 \pm 0.81 / \mathrm{min} / \mathrm{m}^{2}\right)$ lower without anaesthesia.

\section{Follow-up}

Follow-up data were obtained in all 50 patients. Over a followup of $220 \pm 179$ days, 12 (24\%) patients had an adverse cardiovascular event (death $(n=7)$, open-heart surgery for $M R(n=4)$, or hospitalisation for CHF $(n=7)$ ). Figure 3 shows changes in NYHA functional status and MR severity on follow-up. At 3 months after MVR, a decrease was noted in LV end-diastolic volume (from $163 \pm 111$ to $143 \pm 99 \mathrm{ml}, \mathrm{p}=0.01$ ) and LV endsystolic volume (from $98 \pm 102$ to $68 \pm 86 \mathrm{ml}, \mathrm{p}=0.002$ ). However, no significant changes were noted in LV ejection fraction (from $47 \pm 18 \%$ to $47 \pm 16 \%, \mathrm{p}=0.61$ ) or LA diameter (from $54 \pm 14$ to $52 \pm 9 \mathrm{~mm}, \mathrm{p}=0.83$ ).

The area under the receiver operating characteristics curve with respect to outcome (death, open-heart surgery for $M R$, hospitalisation for CHF) was large for changes in PCWP v-wave, mPCWP and mPAP but small for changes in CI (figure 4). A reduction in $\mathrm{mPCWP}$ by $1 \mathrm{~mm} \mathrm{Hg}$ and in PCWP v-wave by $4 \mathrm{~mm} \mathrm{Hg}$ provided the best cut-off point to predict events on

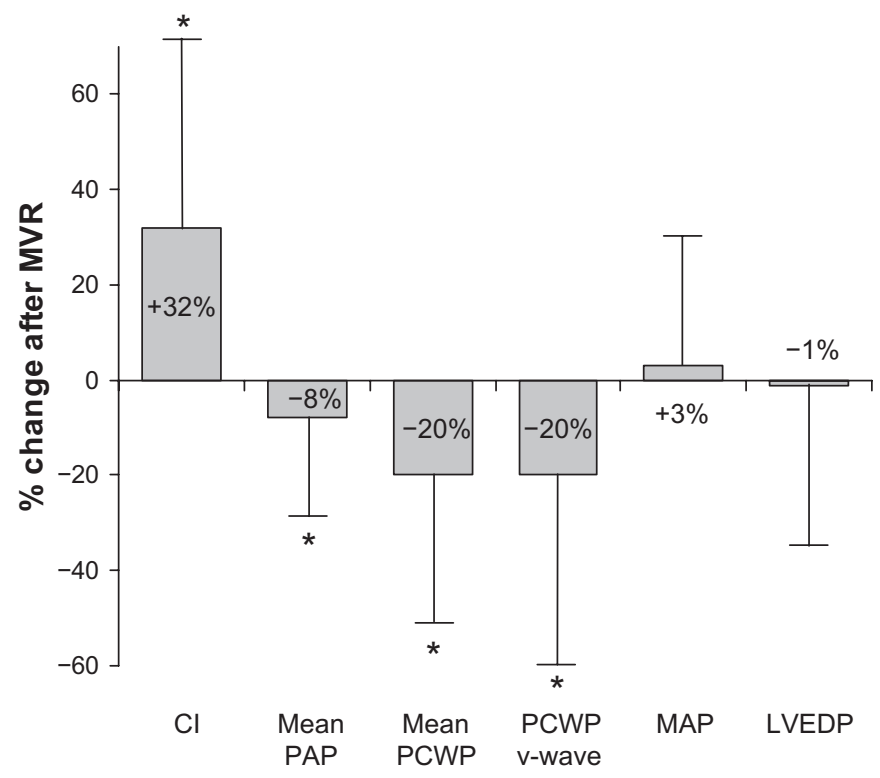

Figure 1 Mean percentage changes in haemodynamic variables after mitral clipping (as a percentage of baselines values). $\mathrm{Cl}$, cardiac index; LVEDP, left ventricular end-diastolic pressure; MAP, mean arterial pressure; MVR, mitral valve repair; $\mathrm{PAP}$, pulmonary artery pressure; PCWP, pulmonary capillary wedge pressure. ${ }^{*} p<0.05$. follow-up. Similarly, on univariate Cox regression analysis, predictors of cardiac outcome were changes in PCWP v-wave $(\mathrm{HR}=1.03$; 95\% CI 1.01 to $1.06 ; \mathrm{p}=0.005)$, mPCWP $(\mathrm{HR}=1.05$; $95 \%$ CI 1.01 to $1.08, \mathrm{p}=0.004)$ and $\mathrm{mPAP}(\mathrm{HR}=1.05 ; 95 \% \mathrm{CI}$ 1.01 to $1.09, \mathrm{p}=0.008)$.

\section{DISCUSSION}

This study documents an improvement in haemodynamic profile immediately after percutaneous edge-to-edge MVR with the MitraClip system measured by right heart catheterisation in a series of 50 consecutive patients with predominantly functional MR. Mitral clipping resulted in an increase in CI by $32 \%$ and a reduction in mean PCWP and PAP by $20 \%$ and $8 \%$, respectively. Most importantly, the acute haemodynamic improvement was associated with a favourable mid-term outcome. At 3 months, 79\% of patients were in NYHA functional class $\mathrm{I}-\mathrm{II}$ and $72 \%$ had $1+$ to $2+$ grade $\mathrm{MR}$, supporting the validity and durability of acute haemodynamic improvements. A decrease in PCWP v-wave, mPCWP and mPAP immediately after mitral valve clipping was predictive of a favourable cardiac outcome over a mean follow-up of 220 days with lower risk for death, open surgery for $\mathrm{MR}$ and hospitalisation for $\mathrm{CHF}$. Additionally, a significant improvement in NYHA functional class after 3 months was seen, suggesting that the acute lowering of pulmonary pressures was preserved over a longer period and translated into an improved functional capacity.

Our results are in line with haemodynamic data obtained from the pivotal EVEREST trial, although subtle differences should be noted. In their recent publication, Siegel and colleagues ${ }^{13}$ report a similar reduction in SVR and an increase in CI. However, in contrast to our findings, they found a significant reduction of LVEDP while PCWP (mean and v-wave) and PAP were unchanged after percutaneous MVR. These discrepancies are probably related to significant differences in patients' baseline characteristics. While the EVEREST trial included mainly patients with degenerative $M R$ and had very stringent inclusion criteria for valve morphology and LV dimensions and function, ${ }^{7} 81113$ our study population consisted predominantly of patients with functional MR and impaired LV function. Therefore, we hypothesise that eliminating regurgitant flow into the left atrium and the pulmonary veins reduced pulmonary pressures, while the acute increase in afterload (by removing the low-impedance regurgitant flow) imposed on a compromised left ventricle was responsible for the lack of changes in LVEDP. Nonetheless, CI increased after percutaneous MVR, indicating that the positive effects of removing regurgitant flow outweighed the potentially harmful increase in afterload, and thereby improved cardiac forward output. Of note, none of the patients with successful MitraClip implantation experienced an acute low output state after the procedure.

While MVR is an established treatment for patients with degenerative $M R$, its role in the management of functional $M R$ is matter of continuing debate. ${ }^{18}$ In patients with ischaemic cardiomyopathy or with acute coronary syndrome, functional $\mathrm{MR}$ is an independent predictor of cardiovascular death with a relative risk varying from 1.5 to $7.5 .{ }^{19-22}$ However, until now, there has been a lack of evidence to suggest that correction of functional MR may reduce cardiovascular events. ${ }^{23} 24$ At present, the AHA/ACC ${ }^{14}$ and the European guidelines ${ }^{25}$ have given IIb recommendations for surgical repair of functional MR in patients who are unsuitable for coronary revascularisation. This is explained by the understandable concern obtained from observation of surgical series that the potential increase in LV afterload, owing to the elimination of the low-impedance 
Figure 2 Exemplary case showing haemodynamic changes after mitral valve repair (MVR) in an 87-year-old man with flail anterior leaflet and severe $(4+)$ mitral regurgitation (MR). ( $A, B)$ Fluoroscopy shows implantation of two MitraClips ( $B$, arrow). Acute procedural success is documented by a reduction of MR from $(4+)(C$, arrow) to $(1+)(D$, arrow) on transoesophageal echocardiography. Pressure tracings $(E, F)$ show a reduction in mean pulmonary capillary wedge pressure (PCWP) (from $20 \mathrm{~mm} \mathrm{Hg}$ to $8 \mathrm{~mm} \mathrm{Hg}$ ) and v-wave (from $42 \mathrm{~mm} \mathrm{Hg}$ to $11 \mathrm{~mm} \mathrm{Hg}$ ) with MVR. Consistently, the cardiac index increased from $1.85 \mathrm{l} / \mathrm{min} / \mathrm{m}^{2}$ to $4.38 \mathrm{l} / \mathrm{min} / \mathrm{m}^{2}$. Real-time pressure volume loops $(G, H)$ obtained with a conductance catheter (CD Leycom, Zoetemeer,

Netherlands) document the increase in afterload leading to an upward and rightward displacement of the end-systolic pressure (ESP) volume point (blue dot), and a reduction in ejection fraction (EF). Of note, end-diastolic pressures (EDPs) are similar (and at low level) before and after MVR, indicating that the reduction in PCWP results from removal of the regurgitant burden on the pulmonary circulation rather than reductions in left ventricular filling pressures. EDV, end-diastolic volume; ESV, end-systolic volume.

\section{Before MVR}
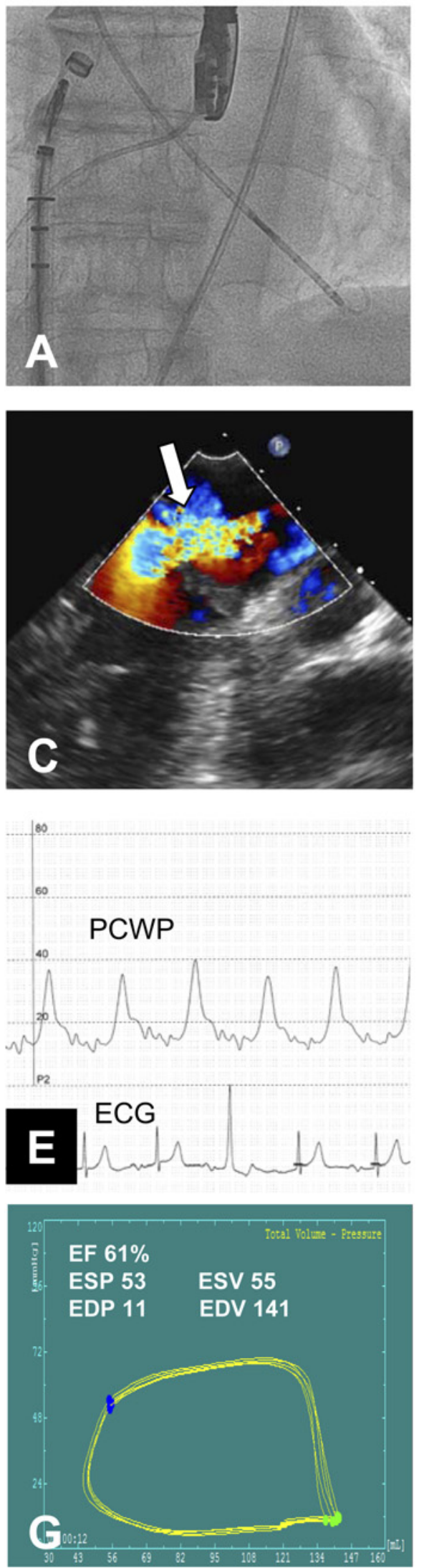

\section{After MVR}
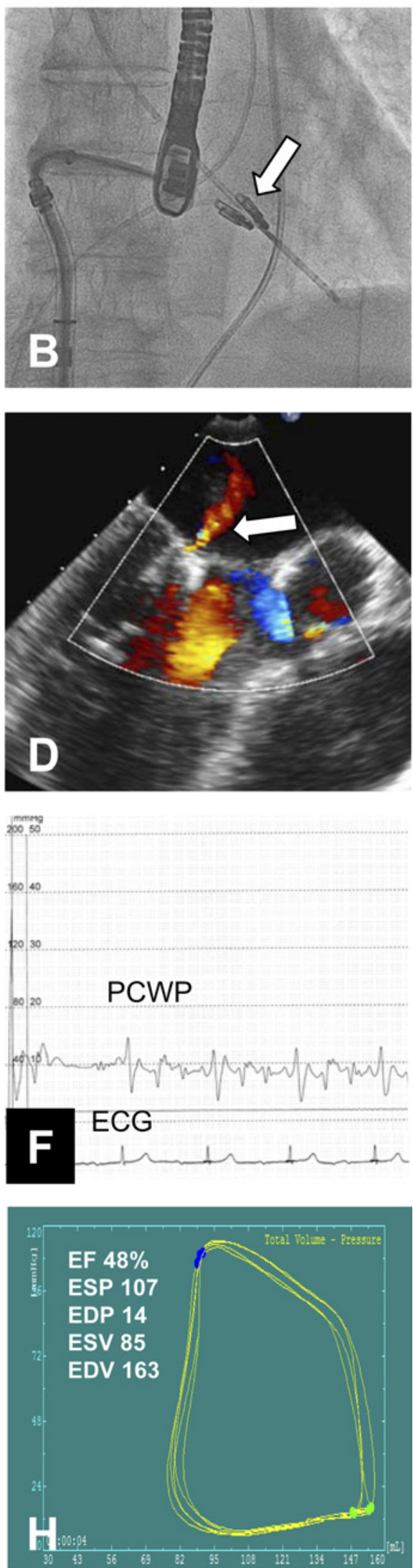
Figure 3 Improvement in New York Heart Association (NYHA) functional class $(A)$ and mitral regurgitation (MR) severity (B) at 3 months after percutaneous mitral valve repair (MVR).
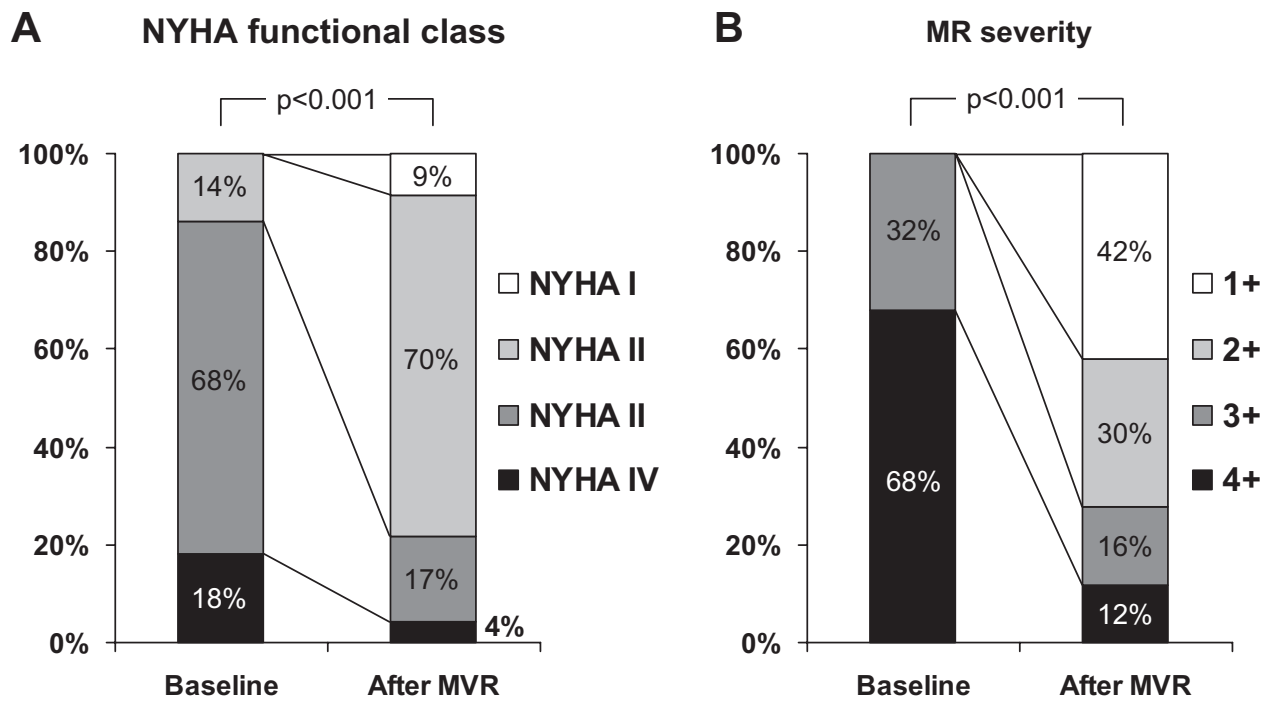

regurgitant flow into the left atrium, may affect ventricular performance and result in an acute postoperative low-output state. ${ }^{1}$ However, percutaneous MVR provides a unique pathophysiological model for assessing haemodynamic changes of $M R$ reduction on LV performance, eliminating any confounding effects from cardiopulmonary bypass. Our results in a population with predominantly functional MR, reduced LV function and dilated left ventricle argue against a detrimental effect of $M R$ reduction, and support the use of percutaneous MVR.

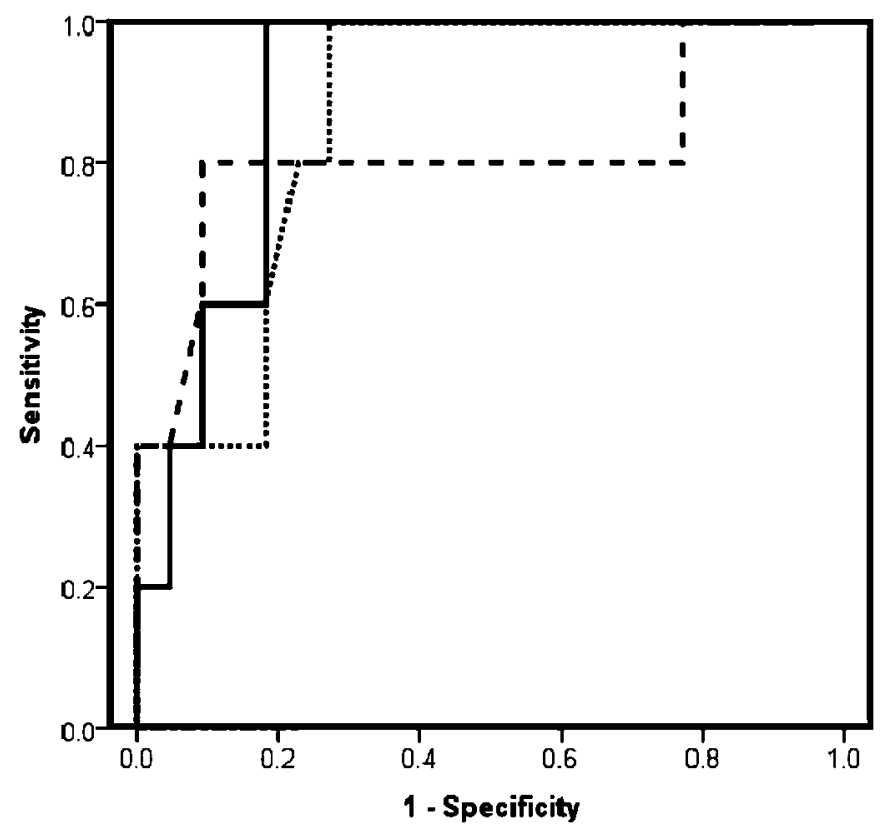

$\begin{array}{llc} & & \text { AUC }(95 \% \mathrm{Cl}) \\ \text {....... } & \text { mPCWP decrease } & 0.90(0.78 \text { to } 1.00) \\ \text { - - }- & \text { mPAP decrease } & 0.87(0.72 \text { to } 1.00) \\ & & 0.81(0.55 \text { to } 1.00)\end{array}$

Figure 4 Receiver operating characteristics analysis for haemodynamic variables to predict events (death, open-heart surgery for mitral regurgitation, hospitalisation for congestive heart failure) with their respective area under the curve (AUC) given below. mPAP, mean pulmonary artery pressure; PCWP, pulmonary capillary wedge pressure.
Larger trials with more homogeneous populations will be needed to confirm these results.

Finally, the use of transoesophageal echocardiography to assess suitability for percutaneous MVR should be emphasised. Although there is no objective data to suggest a benefit of $3 \mathrm{D}$ over 2D echo, 3D visualisation (by 'live 3D' or '3D zoom' modes) may provide useful information on mitral valve anatomy and allows the orientation of the device in 3D. Additionally, the unique ability of $3 \mathrm{D}$ echocardiography to provide simultaneous views of the mitral valve (so called 'xPlane view') is crucial for adequate clip placement. Finally, 'full volume colour Doppler' loops are helpful for determining the presence and location of residual jets after clip placement and facilitate correct placement of additional clips.

\section{Limitations}

Given the novelty of percutaneous MVR this report included a small number of patients. The limited number of patients also precluded a multivariate analysis to identify predictors for a haemodynamic improvement after MVR. Therefore, this report should be considered as preliminary and hypothesisgenerating and should encourage larger studies to confirm our results.

Right heart catheterisation was performed under general anaesthesia. This led to underestimation of pulmonary artery and capillary wedge pressure and lowering of LV pre- and afterload compared with the conscious state. A comparison with baseline variables obtained in a subgroup of patients indicates marked changes in haemodynamic profiles induced by general anaesthesia (table 4). Additionally, hyperoxygenation resulted in higher mixed venous oxygen saturations and, consequently, higher values for $\mathrm{CO}$ and $\mathrm{CI}$ calculated by the Fick method than would by expected for this population. An alternative would have been to perform thermodilution measurement of $\mathrm{CO}$. However, the high prevalence of significant tricuspid regurgitation would have affected the validity of results. Additionally, although oximetry was performed with the trans-septal sheath in place, left-to-right shunting from iatrogenic atrial septal defect may have artificially increased CI values.

This study assessed only acute changes in haemodynamic profile. We did not repeat right heart catheterisation on follow-up as this would have required subjecting patients to a repeat invasive study, which was not part of the study 
protocol. Therefore it is unclear whether the observed haemodynamic improvements are maintained over a longer period of time.

Finally, we did not perform calculations of effective regurgitant orifice area using the proximal isovelocity surface area method before and after MVR. This method has not been validated for double-orifice mitral valves and therefore the validity of results after MVR would have been questionable.

\section{CONCLUSION}

MVR with the Evalve MitraClip system acutely lowers PCWP by $20 \%$ and PAP by $8 \%$, and increases CI by $32 \%$ in a heterogeneous population with degenerative and functional $M R$.

\section{Competing interests None.}

Patient consent Obtained.

Ethics approval This study was conducted with the approval of the institutional review board of the University Hospital Zurich.

Contributors We herewith state that all authors have contributed significantly to (1) conception and design, or analysis and interpretation of data; (2) drafting the article or revising it critically for important intellectual content; (3) final approval of the version to be published.

Provenance and peer review Not commissioned; externally peer reviewed.

\section{REFERENCES}

1. Enriquez-Sarano M, Schaff HV, Orszulak TA, et al. Valve repair improves the outcome of surgery for mitral regurgitation. A multivariate analysis. Circulation 1995; 91:1022-8.

2. Akins CW, Hilgenberg $A D$, Buckley MJ, et al. Mitral valve reconstruction versus replacement for degenerative or ischemic mitral regurgitation. Ann Thorac Surg 1994;58:668-75; discussion 75-6.

3. Vassileva CM, Boley T, Markwell S, et al. Meta-analysis of short-term and longterm survival following repair versus replacement for ischemic mitral regurgitation. Eur J Cardiothorac Surg 2011;39:295-303.

4. Magne J, Girerd N, Senechal M, et al. Mitral repair versus replacement for ischemic mitral regurgitation: comparison of short-term and long-term survival. Circulation 2009;120:S104-11.

5. St Goar FG, Fann Jl, Komtebedde J, et al. Endovascular edge-to-edge mitral valve repair: short-term results in a porcine model. Circulation 2003;108:1990-3.

6. Alfieri 0, Maisano F, De Bonis M, et al. The double-orifice technique in mitral valve repair: a simple solution for complex problems. J Thorac Cardiovasc Surg 2001;122:674-81

7. Feldman $\mathbf{T}$, Kar $\mathrm{S}$, Rinaldi $\mathrm{M}$, et al. Percutaneous mitral repair with the MitraClip system: safety and midterm durability in the initial EVEREST (Endovascular Valve Edge-to-Edge REpair Study) cohort. J Am Coll Cardiol 2009;54:686-94.
8. Feldman T, Wasserman HS, Herrmann $\mathrm{HC}$, et al. Percutaneous mitral valve repair using the edge-to-edge technique: six-month results of the EVEREST Phase I Clinical Trial. J Am Coll Cardiol 2005;46:2134-40.

9. Franzen 0, Baldus S, Rudolph V, et al. Acute outcomes of MitraClip therapy for mitral regurgitation in high-surgical-risk patients: emphasis on adverse valve morphology and severe left ventricular dysfunction. Eur Heart J 2010:31:1373-81.

10. Tamburino C, Ussia GP, Maisano F, et al. Percutaneous mitral valve repair with the MitraClip system: acute results from a real world setting. Eur Heart J 2010;31:1382-9.

11. Feldman T, Foster E, Glower DG, et al. Percutaneous repair or surgery for mitral regurgitation. N Engl J Med 2011;364:1395-406.

12. Zoghbi WA, Enriquez-Sarano M, Foster E, et al. Recommendations for evaluation of the severity of native valvular regurgitation with two-dimensional and Doppler echocardiography. J Am Soc Echocardiogr 2003;16:777-802.

13. Siegel RJ, Biner S, Rafique AM, et al. The acute hemodynamic effects of MitraClip therapy. J Am Coll Cardiol 2011:57:1658-65.

14. Bonow RO, Carabello BA, Kanu C, et al. ACC/AHA 2006 guidelines for the management of patients with valvular heart disease: a report of the American College of Cardiology/American Heart Association Task Force on Practice Guidelines (writing committee to revise the 1998 Guidelines for the Management of Patients With Valvular Heart Disease): developed in collaboration with the Society of Cardiovascular Anesthesiologists: endorsed by the Society for Cardiovascular Angiography and Interventions and the Society of Thoracic Surgeons. Circulation 2006:114:e84-231.

15. Roques F, Nashef SA, Michel P, et al. Risk factors and outcome in European cardiac surgery: analysis of the EuroSCORE multinational database of 19030 patients. Eur J Cardiothorac Surg 1999;15:816-22; discussion 22-3.

16. Biaggi P, Gruner C, Jedrzkiewicz $\mathrm{S}$, et al. Assessment of mitral valve prolapse by 3D TEE angled views are key. JACC Cardiovasc Imaging 2011:4:94-7.

17. Selzer A, Sudrann RB. Reliability of the determination of cardiac output in man by means of the Fick principle. Circ Res 1958;6:485-90.

18. Pierard LA, Carabello BA. Ischaemic mitral regurgitation: pathophysiology, outcomes and the conundrum of treatment. Eur Heart $J$ 2010;31:2996-3005

19. Lamas GA, Mitchell GF, Flaker GC, et al. Clinical significance of mitral regurgitation after acute myocardial infarction. Survival and Ventricular Enlargement Investigators. Circulation 1997; 96:827-33.

20. Bursi F, Enriquez-Sarano M, Nkomo VT, et al. Heart failure and death after myocardial infarction in the community: the emerging role of mitral regurgitation. Circulation 2005;111:295-301.

21. Lehmann KG, Francis CK, Dodge HT. Mitral regurgitation in early myocardial infarction. Incidence, clinical detection, and prognostic implications. TIMI Study Group. Ann Intern Med 1992;117:10-17.

22. Persson A, Hartford M, Herlitz J, et al. Long-term prognostic value of mitral regurgitation in acute coronary syndromes. Heart 2010;96:1803-8.

23. Wu AH, Aaronson KD, Bolling SF, et al. Impact of mitral valve annuloplasty on mortality risk in patients with mitral regurgitation and left ventricular systolic dysfunction. J Am Coll Cardiol 2005;45:381-7.

24. Trichon BH, Glower DD, Shaw LK, et al. Survival after coronary revascularization, with and without mitral valve surgery, in patients with ischemic mitral regurgitation. Circulation 2003;108 Suppl 1:I103-10.

25. Vahanian A, Baumgartner $\mathrm{H}, \mathrm{Bax} \mathrm{J}$, et al. Guidelines on the management of valvula heart disease: The Task Force on the Management of Valvular Heart Disease of the European Society of Cardiology. Eur Heart J 2007;28:230-68. 